\title{
The Role of Sharp Dissection in Nipple-Sparing Mastectomy: A Safe Procedure with No Necrosis of the Nipple-Areolar Complex
}

This article was published in the following Dove Press journal: Cancer Management and Research

\author{
Ci-Qiu Yang* \\ Fei Ji* \\ Hong-Fei Gao \\ Liu-Lu Zhang \\ Mei Yang \\ Teng Zhu \\ Min-yi Chen \\ Jie-qing Li \\ Kun Wang
}

Department of Breast Cancer, Cancer Center, Guangdong Provincial People's Hospital \& Guangdong Academy of

Medical Sciences, Guangzhou, People's

Republic of China

*These authors contributed equally to this work
Correspondence: Kun Wang

Department of Breast Cancer, Cancer Center, Guangdong Provincial People's Hospital \& Guangdong Academy of Medical Sciences, Guangzhou 510080 , People's Republic of China

$\mathrm{Tel} / \mathrm{Fax}+86$ 20-838278I 2-80420

Email gzwangkun@126.com
Background: Nipple-sparing mastectomy (NSM) is becoming increasingly accepted as a treatment for breast disease; however, nipple-areolar complex (NAC) necrosis, a frequent severe postoperative complication, inhibits the popularity of this procedure. This study reports the technical aspects and short-term postoperative outcomes of NSM.

Methods: A single-center, retrospective review of 110 patients treated with NSM at our institution from November 2015 to September 2018 was performed. The primary outcome was the incidence of NAC necrosis.

Results: A total of 130 NSMs performed on 110 patients were included in our study. Median patient age was 42 years. We performed a sharp dissection by using a scalpel, raising 3-5 mm thick flaps, and continuing onto the undersurface of the NAC. None of the 110 patients appeared to have NAC necrosis or mastectomy skin flap necrosis. However, discoloration or ischemia of the NAC with eschar formation presented between postoperative days 3 and 7 in six nipples; four nipples were ischemic, and two were discolored. No infection was detected in any of the 110 patients. All NACs were intact after an average follow-up of 30 months, and no local or systemic recurrence was detected in those breast cancer cases.

Conclusion: NSM can be safely performed in properly selected patients. Nipple necrosis was avoided using a special surgical technique, and other complications occurred at an acceptable rate.

Keywords: nipple-sparing mastectomy, breast cancer, nipple-areolar complex, sharp dissection

\section{Introduction}

The progress of mastectomy techniques has advanced from radical mastectomy to skin-sparing mastectomy (SSM) and then to nipple-sparing mastectomy (NSM). ${ }^{1,2}$ NSM combines SSM with preservation of the nipple-areolar complex (NAC), which improves reconstructive and aesthetic outcomes, leading to higher psychosocial and sexual well-being compared to nipple reconstruction. ${ }^{3-9}$ NSM has been demonstrated to have an equivalent oncological safety profile as that of radical mastectomy, and preserving the NAC does not increase local recurrence or survival chances. $^{10-13}$

During the past decade, NSM has increased remarkably for women with breast cancer or those undergoing risk-reducing contralateral or bilateral prophylactic mastectomy. ${ }^{3,4}$ However, there is no universal consensus on the indications for 
optimal patient selection, although several factors, including tumor size, tumor-to-nipple distance, negative axillary node status, absence of lymphovascular invasion, skin involvement, inflammatory cancer, and a negative margin beneath the nipple have been suggested. ${ }^{14-21}$

The most severe postoperative complication is NAC necrosis. Several studies have described patients who lost part of their nipple following NSM. ${ }^{22}$ In our institution, we previously performed the dissection between the subcutaneous tissue and the glandular tissue using an electrotome on 20 patients between March 2015 and August 2015; however, four nipples became ischemic, two became discolored and two became necrotic, and the rate of complications was $40 \%$. Then, we improved the surgical technique to protect the blood supply to the NAC, and none of the patients lost their nipples. Here, we describe the initial experience of NSM at the breast center of Guangdong General Hospital from November 2015 to September 2018, including the operative procedure, shortterm postoperative outcomes, and a successful technique for preserving the NAC.

\section{Methods}

\section{Patients and Methods}

This study was a retrospective review of 110 patients who underwent 130 NSM procedures at the breast center of Guangdong General Hospital from November 2015 to September 2018. Patient demographics, neo-adjuvant therapy, and reconstructive outcomes, including mastectomy flap necrosis, NAC necrosis, and infection among other variables were abstracted from our departmental and clinical records.

\section{Surgical Technique}

All patients underwent NSM via a radial incision if the retro-areolar biopsy pathology was positive for carcinoma because of the technical similarity to SSM. The incision was extended to remove the nipple or NAC easily rather than making a second incision.

NSM was performed via radial incision without taking any skin. A mixed liquid of $500 \mathrm{~mL}$ normal saline and $1 \mathrm{mg}$ epinephrine was injected subcutaneously into the breast. Then, we performed a sharp dissection using a scalpel, raising 3-5 $\mathrm{mm}$ thick flaps, and continuing onto the undersurface of the NAC (Figure 1). All patients underwent a retro-areolar biopsy with a permanent section and the evaluation was conducted intraoperatively by a breast

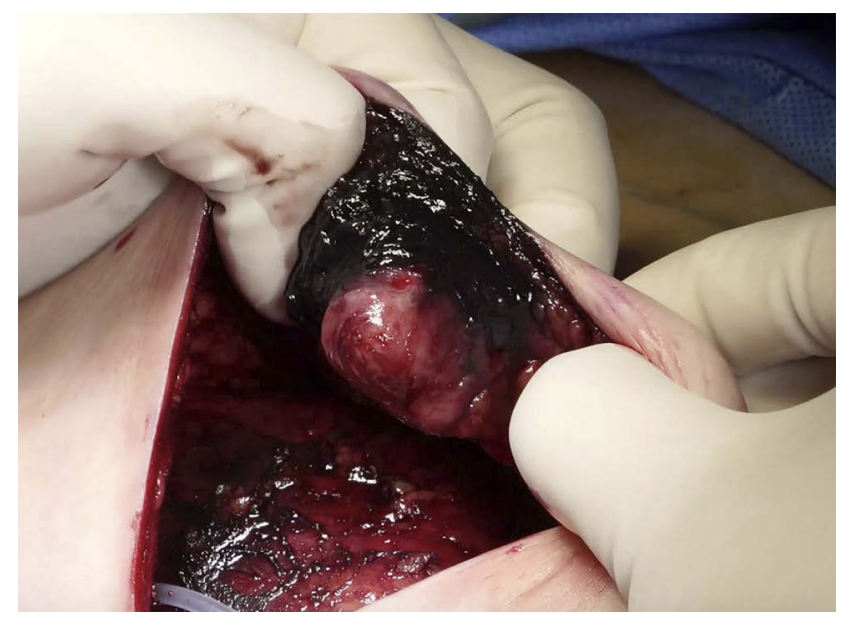

Figure I Tissue underneath the nipple-areolar complex (NAC).

pathologist. We took care to remove the breast at the level of the breast capsule to maximize the reduction in glandular volume and minimize trauma to the breast skin envelope. We tried to remove all of the breast tissue underneath the NAC based on a subcutaneous mastectomy, which intentionally retains retro-areolar breast tissue due to concerns about nipple ischemia. Minimal tissue was left $(<0.5 \mathrm{~cm})$ under the NAC when treating patients with breast cancer. Drains were universally left in the residual cavity of all patients, and we did not use prophylactic antibiotics.

All patients in this study underwent NSM for breast cancer treatment or prophylaxis. Four women received a direct implant, whereas 90 women received tissue expander reconstruction to be exchanged for the permanent implant at a second stage; 7 patients only underwent NSM, 83 women with breast cancer underwent NSM and sentinel lymph node biopsy, and 16 patients underwent axillary lymph node dissection because of a positive sentinel lymph node biopsy.

\section{Ethics Statement}

This study was approved by an independent ethical institutional review board at Guangdong Provincial People's Hospital and was carried out in accordance with the principles of the Declaration of Helsinki. It is a retrospective study and does not require the patient's informed consent.

\section{Results}

We identified 110 patients who underwent 130 nipplesparing procedures at the breast center of Guangdong General Hospital from November 2015 to September 2018. The median age at the time of the operation was 42 years 
(range, 24-70 years). The median follow-up period was 30 months (range, 12-46 months). Histology and staging for the patients with NSM are detailed in Table 1.

Eighty-three women with breast cancer underwent therapeutic NSM procedures; 61 (73.49\%) for invasive breast cancer and 22 (26.51\%) for ductal carcinoma in situ. Among the breast cancers, $50(60.24 \%)$ were $\leq 2 \mathrm{~cm}, 31(37.35 \%)$ were $\leq 5 \mathrm{~cm}$ but $>2 \mathrm{~cm}$, and $2(2.41 \%)$ were $>5 \mathrm{~cm}$. Mean tumor size was $1.8 \mathrm{~cm}$ (range $0.4-7.4 \mathrm{~cm}$ ).

All retroareolar tissue specimens were negative, and no invasive cancers were detected in the prophylactic NSM specimens. Among the invasive cancers, axillary lymph node metastases were detected in 16 patients $(26.23 \%)$, while the other 45 patients $(73.77 \%)$ were lymph nodenegative. Four received direct implants (Figure 2), while 90 received tissue expander reconstruction to be exchanged for a permanent implant at the second stage (Figure 3 ).

Table I Patients' Characteristics

\begin{tabular}{|l|l|l|}
\hline Characteristic & $\mathbf{n} / \mathbf{N}$ & $\%$ \\
\hline Median age at surgery: Years (Range) & $42(24-70)$ & \\
\hline Disease & & \\
Breast cancer & $83 / 110$ & $75.45 \%$ \\
Flat epithelial atypia & $2 / 110$ & $1.82 \%$ \\
Papilloma & $4 / 110$ & $3.64 \%$ \\
Hyperplastic pain & $7 / 110$ & $6.36 \%$ \\
Repeated multiple fibroma & $1 / 110$ & $0.91 \%$ \\
Serous mastitis & $1 / 110$ & $0.91 \%$ \\
Side effect after injecting Amazingel & $3 / 110$ & $2.73 \%$ \\
Gynecomastia & $9 / 110$ & $8.18 \%$ \\
\hline Histologic tumor type & & \\
Invasive & $61 / 83$ & $73.49 \%$ \\
DCIS & $22 / 83$ & $26.51 \%$ \\
\hline Tumor size & & \\
$\leq 2$ cm & $50 / 83$ & $60.24 \%$ \\
$\leq 5 \mathrm{~cm}$ and $>2$ cm & $31 / 83$ & $37.35 \%$ \\
$>5 \mathrm{~cm}$ & $2 / 83$ & $2.41 \%$ \\
\hline Immunohistochemistry & & \\
ER/PR positive & $46 / 61$ & $75.41 \%$ \\
Her-2 positive & $16 / 61$ & $26.23 \%$ \\
Triple negative & $4 / 61$ & $6.56 \%$ \\
\hline Therapy before operation & & \\
Neoadjuvant chemotherapy & $5 / 61$ & $29.51 \%$ \\
Neoadjuvant endocrine therapy & & \\
\hline Lymph node & & \\
Positive & & \\
Negative & & \\
\hline & & \\
\hline
\end{tabular}

The most severe postoperative complication is NAC necrosis, but none of the 110 patients developed NAC necrosis or mastectomy skin flap necrosis. The most common postoperative complication was discoloration or ischemia of the NAC with eschar formation presenting between postoperative days 3 and 7 in six nipples (4.62\%); four nipples were ischemic and two were discolored. The decrustation of the six patients who had nipple ischemia with eschar formation occurred 3 months after the operation. No infections occurred among the 110 patients.

During the median follow-up periods of 30 months (range, 12-46 months), the 130 nipple-areolar complexes remained intact, and no local or systemic recurrence was observed in those cases treated for cancer. No late complications, such as capsular contraction or shifts in prostheses, occurred.

\section{Discussion}

The use of NSM has increased significantly in recent years and became a standard procedure for the treatment of cancer and for risk-reducing prophylactic mastectomy. ${ }^{3,23}$ NSM maintains equivalent oncological outcomes and provides superior satisfaction for the patient compared to traditional mastectomy. However, postoperative necrosis of the NAC is a severe clinical complication; thus, how to protect the blood supply of NAC has become a pivotal issue.

Several studies have demonstrated that blood supply to the NAC originates from a complex vascular network, including the lateral thoracic artery, intercostal artery, internal thoracic artery and thoracoacromial artery. ${ }^{24-27}$ This vascular network that supports the blood supply to the NAC is divided into a subcutaneous vascular network, which is under the skin but above the glandular tissue, whereas the other part of the network includes perforating branches that run vertically through the glandular tissue. The perforating branches are cut off during NSM, and only the subcutaneous vascular network is reserved for the blood supply to the NAC. Several studies have described patients losing part of their nipples following NSM. The rates of nipple loss are $0-53 \%$ for tumor involvement of the nipple and $2.9-10 \%$ for ischemia or necrosis of the NAC. ${ }^{5}$ The reasons for this wide range are complex, but surgical technique played a role in the complication. There is still no standard surgical technique for NSM and the procedure varies widely among surgeons. Previous studies have argued that sharp dissection could create more trauma to the skin flaps and increase necrosis of the NAC, ${ }^{28,29}$ However, others have suggested that sharp 

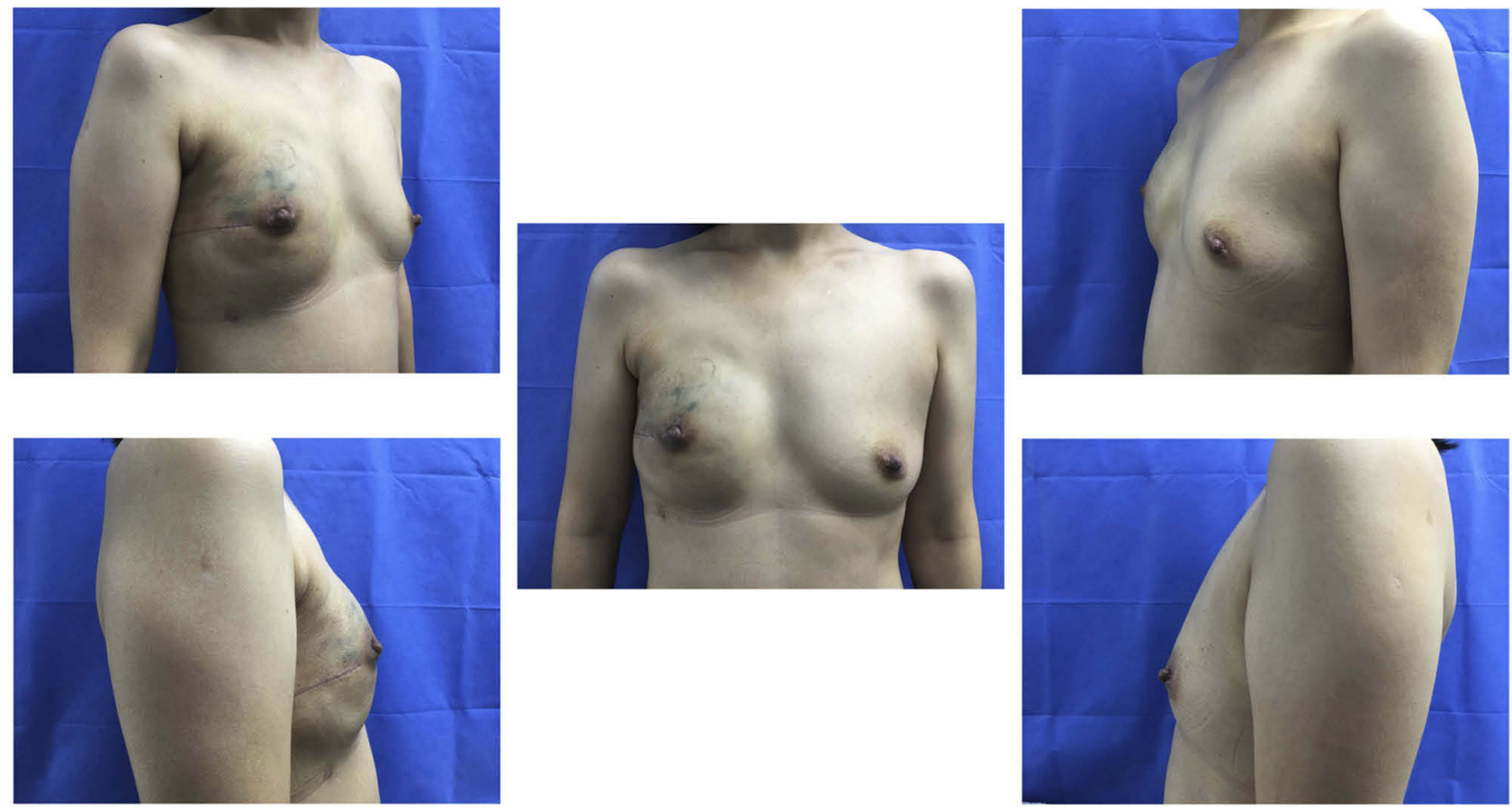

Figure 2 Right breast cancer with nipple-sparing mastectomy and direct implant reconstruction: postoperative view at 3 months.
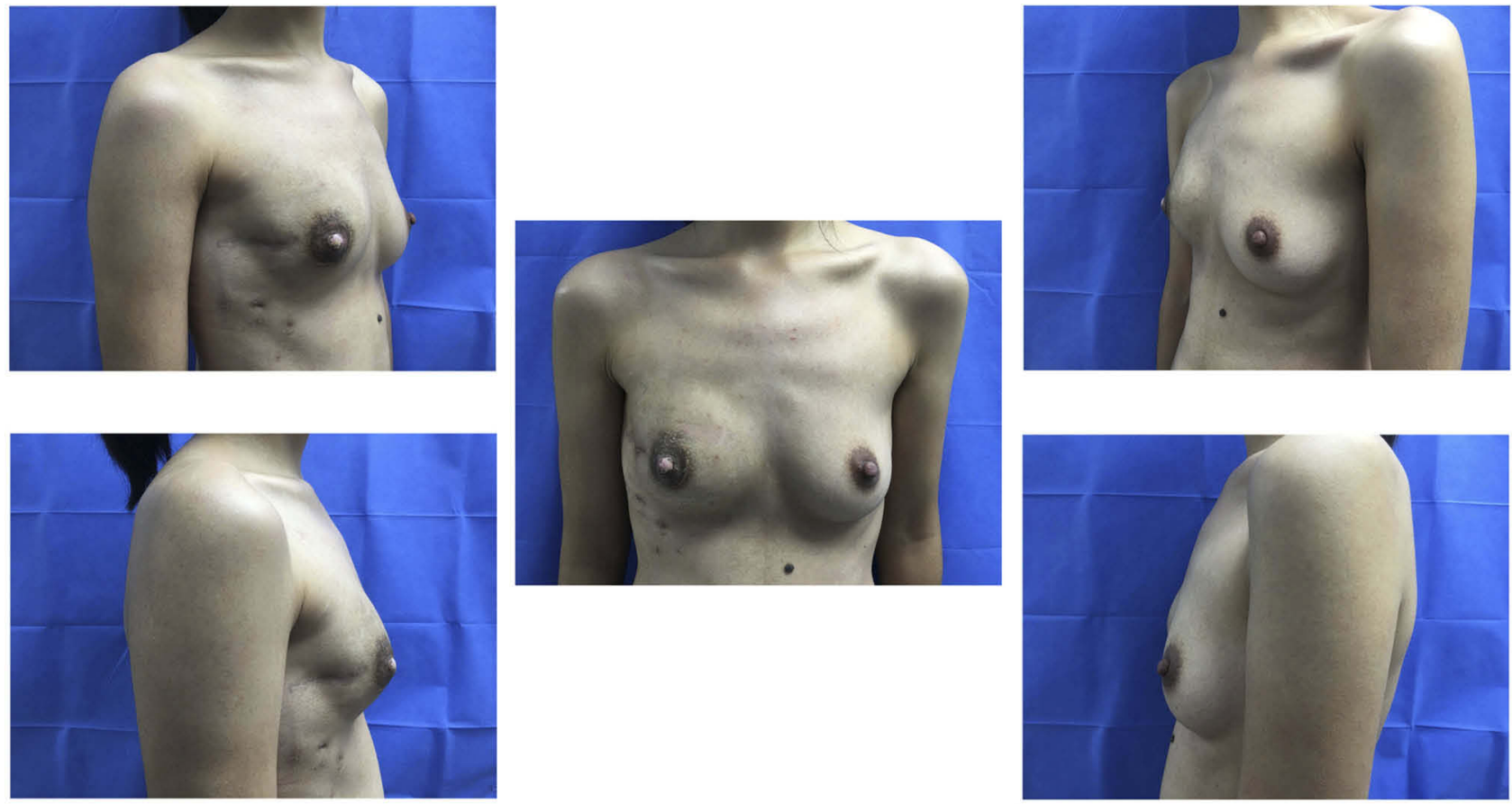

Figure 3 Right breast cancer with nipple-sparing mastectomy and tissue expander reconstruction.

dissection could protect the blood supply of the NAC, resulting in a lower rate of necrosis. ${ }^{6}$ NSM represented approximately $10 \%$ of all mastectomies performed in our department during the study period and became a standard surgical procedure for breast disease in our institution. We performed a sharp dissection using a scalpel with minimal electrocautery, raising 3-5 $\mathrm{mm}$ thin subdermal flaps, and continuing onto the undersurface of the NAC to protect the subcutaneous vascular network. The NAC was successfully preserved in all cases, and the incidence of nipple loss was 
zero. Discoloration or ischemia with eschar formation of the NAC represented $4.62 \%$ of all cases, which eventually developed intact and well-healed scars 2 months after the operation. We argue that the low incidence of complications was due to our surgical technique of using sharp dissection with minimal electrocautery, which protected the blood supply to the NAC.

To the best of our knowledge, there is still no universal consensus on the selection criteria for NSM. Studies have described some relative selection criteria: tumor size $<3 \mathrm{~cm}$, distance from the tumor to the nipple $<2 \mathrm{~cm}$, and negative lymph nodes, which promises these patients the lowest risk of occult NAC involvement. ${ }^{3,14-18,30}$ However, in our center, patients were given the option of NSM with looser selection criteria depending on a physical examination and magnetic resonance imaging (MRI). We did not perform NSM for those patients with suspicion of carcinoma-related NAC by clinical assessment or MRI. All frozen retro-areolar biopsy sections were negative. However, we have no hard cutoff for tumor size or lymph nodes. In our study, median tumor size was $1.8 \mathrm{~cm}$ (range, $0.4-7.4 \mathrm{~cm}$ ), and 16 patients $(26.23 \%$ ) had positive lymph node metastases.

Chemotherapy is a significant treatment for most breast cancer. These cytotoxic agents rapidly kill proliferating cells $^{31-35}$ but can impair regenerative cells, which are responsible for wound healing, and may increase the complication rates in the setting of breast reconstructive surgery. ${ }^{35-38}$ However, based on our data, none of the patients with breast cancer showed poor wound healing, NAC discoloration, or ischemia. We demonstrated that NSM can be safe if offered to those who have received neoadjuvant chemotherapy or endocrine therapy.

Previous studies have reported that preserving the NAC does not increase local recurrence or survival chances. ${ }^{10-13}$ Our study also found no locoregional recurrence or distant recurrence with a median follow-up of 30 months (range: 12-46 months). These results are promising; however, the short follow-up period was a limitation of our study, so a longer follow-up period of this cohort is necessary.

\section{Acknowledgments}

The authors acknowledge the patients who had participated the study, especially those who have given their permission for us to publish their photos in this paper. This study was supported by grants from "Guangdong Medical Science and Technology Research Fund (grant numbers A2019494 and A2019252)" and "The Doctor Launch Fund of Guangdong Provincial People's Hospital (2018bq04)".

\section{Disclosure}

The authors report no conflicts of interest in this work.

\section{References}

1. Didier F, Radice D, Gandini S, et al. Does nipple preservation in mastectomy improve satisfaction with cosmetic results, psychological adjustment, body image and sexuality? Breast Cancer Res Treat. 2009;118(3):623-633. doi:10.1007/s10549-008-0238-4

2. Didier F, Arnaboldi P, Gandini S, et al. Why do women accept to undergo a nipple sparing mastectomy or to reconstruct the nipple areola complex when nipple sparing mastectomy is not possible? Breast Cancer Res Treat. 2012;132(3):1177-1184. doi:10.1007/ s10549-012-1983-y

3. Agarwal S, Agarwal S, Neumayer L, Agarwal JP. Therapeutic nipple-sparing mastectomy: trends based on a national cancer database. Am J Surg. 2014;208(1):93-98. doi:10.1016/j.amjsurg.2013.09.030

4. de Alcantara Filho P, Capko D, Barry JM, Morrow M, Pusic A, Sacchini VS. Nipple-sparing mastectomy for breast cancer and risk-reducing surgery: the Memorial Sloan-Kettering Cancer Center experience. Ann Surg Oncol. 2011;18(11):3117-3122. doi:10.1245/ s10434-011-1974-y

5. Mallon P, Feron JG, Couturaud B, et al. The role of nipple-sparing mastectomy in breast cancer: a comprehensive review of the literature. Plast Reconstr Surg. 2013;131(5):969-984. doi:10.1097/ PRS.0b013e3182865a3c

6. Endara M, Chen D, Verma K, Nahabedian MY, Spear SL. Breast reconstruction following nipple-sparing mastectomy: a systematic review of the literature with pooled analysis. Plast Reconstr Surg. 2013;132(5):1043-1054. doi:10.1097/PRS.0b013e3182a48b8a

7. Wei $\mathrm{CH}$, Scott AM, Price AN, et al. Psychosocial and sexual well-being following nipple-sparing mastectomy and reconstruction. Breast J. 2016;22(1):10-17. doi:10.1111/tbj.12542

8. Laronga C, Smith P. Nipple-sparing mastectomy: an oncologic and cosmetic perspective. Surg Oncol Clin N Am. 2014;23(3):549-566. doi:10.1016/j.soc.2014.03.013

9. Yoon-Flannery K, DeStefano LM, De La Cruz LM, et al. Quality of life and sexual well-being after nipple sparing mastectomy: a matched comparison of patients using the breast Q. J Surg Oncol. 2018;118(1):238-242. doi:10.1002/jso.25107

10. Burdge EC, Yuen J, Hardee M, et al. Nipple skin-sparing mastectomy is feasible for advanced disease. Ann Surg Oncol. 2013;20 (10):3294-3302. doi:10.1245/s10434-013-3174-4

11. Coopey SB, Tang R, Lei L, et al. Increasing eligibility for nipple-sparing mastectomy. Ann Surg Oncol. 2013;20(10):3218-3222. doi:10.1245/ s10434-013-3152-x

12. Lago V, Maisto V, Gimenez-Climent J, Vila J, Vazquez C, Estevan R. Nipple-sparing mastectomy as treatment for patients with ductal carcinoma in situ: a 10-year follow-up study. Breast J. 2018;24 (3):298-303. doi:10.1111/tbj.2018.24.issue-3

13. Galimberti V, Morigi C, Bagnardi V, et al. Oncological outcomes of nipple-sparing mastectomy: a single-center experience of 1989 patients. Ann Surg Oncol. 2018;25:3849-3857. doi:10.1245/s10434-018-6759-0

14. Spear SL, Willey SC, Feldman ED, et al. Nipple-sparing mastectomy for prophylactic and therapeutic indications. Plast Reconstr Surg. 2011;128(5):1005-1014. doi:10.1097/PRS.0b013e31822b6456

15. Benediktsson KP, Perbeck L. Survival in breast cancer after nipple-sparing subcutaneous mastectomy and immediate reconstruction with implants: a prospective trial with 13 years median follow-up in 216 patients. Eur J Surg Oncol. 2008;34(2):143-148. doi:10.1016/ j.ejso.2007.06.010

16. Mosahebi A, Ramakrishnan V, Gittos M, Collier J. Aesthetic outcome of different techniques of reconstruction following nipple-areola-preserving envelope mastectomy with immediate reconstruction. Plast Reconstr Surg. 2007;119(3):796-803. doi:10.1097/01.prs.0000251999.52374.09 
17. Radovanovic Z, Radovanovic D, Golubovic A, Ivkovic-Kapicl T, Bokorov B, Mandic A. Early complications after nipple-sparing mastectomy and immediate breast reconstruction with silicone prosthesis: results of 214 procedures. Scand J Surg. 2010;99(3):115-118. doi:10.1177/145749691009900302

18. Yang SJ, Eom JS, Lee TJ, Ahn SH, Son BH. Recipient vessel selection in immediate breast reconstruction with free abdominal tissue transfer after nipple-sparing mastectomy. Arch Plast Surg. 2012;39(3):216-221. doi:10.5999/aps.2012.39.3.216

19. Laronga C, Kemp B, Johnston D, Robb GL, Singletary SE. The incidence of occult nipple-areola complex involvement in breast cancer patients receiving a skin-sparing mastectomy. Ann Surg Oncol. 1999;6(6):609-613. doi:10.1007/s10434-999-0609-z

20. Simmons RM, Brennan M, Christos P, King V, Osborne M. Analysis of nipple/areolar involvement with mastectomy: can the areola be preserved? Ann Surg Oncol. 2002;9(2):165-168. doi:10.1007/ BF02557369

21. Vlajcic Z, Zic R, Stanec S, Lambasa S, Petrovecki M, Stanec Z. Nipple-areola complex preservation: predictive factors of neoplastic nipple-areola complex invasion. Ann Plast Surg. 2005;55 (3):240-244. doi:10.1097/01.sap.0000171680.49971.85

22. Ahn SJ, Woo TY, Lee DW, Lew DH, Song SY. Nipple-areolar complex ischemia and necrosis in nipple-sparing mastectomy. Eur J Surg Oncol. 2018;44(8):1170-1176. doi:10.1016/j.ejso.2018.05.006

23. Susini T, Renda I, Giani M, et al. Changing trends in mastectomy and breast reconstruction. Analysis of a Single-Institution Experience Between 2004-2016. Anticancer Res. 2019;39(10):5709-5714.

24. Seitz IA, Nixon AT, Friedewald SM, Rimler JC, Schechter LS. "NACsomes": a new classification system of the blood supply to the nipple areola complex (NAC) based on diagnostic breast MRI exams. J Plast Reconstr Aesthet Surg. 2015;68(6):792-799. doi:10.1016/j.bjps.2015.02.027

25. O'Dey D, Prescher A, Pallua N. Vascular reliability of nipple-areola complex-bearing pedicles: an anatomical microdissection study. Plast Reconstr Surg. 2007;119(4):1167-1177. doi:10.1097/01.prs.00002 54360.98241.dc

26. Nakajima H, Imanishi N, Aiso S. Arterial anatomy of the nipple-areola complex. Plast Reconstr Surg. 1995;96(4):843-845. doi:10.1097/00006534-199509001-00012
27. van Deventer PV. The blood supply to the nipple-areola complex of the human mammary gland. Aesthetic Plast Surg. 2004;28 (6):393-398. doi:10.1007/s00266-003-7113-9

28. Matsen CB, Mehrara B, Eaton A, et al. Skin flap necrosis after mastectomy with reconstruction: a prospective study. Ann Surg Oncol. 2016;23(1):257-264. doi:10.1245/s10434-015-4709-7

29. Peled AW, Foster RD, Ligh C, Esserman LJ, Fowble B, Sbitany H. Impact of total skin-sparing mastectomy incision type on reconstructive complications following radiation therapy. Plast Reconstr Surg. 2014;134(2):169-175. doi:10.1097/PRS.0000000000000386

30. Krajewski AC, Boughey JC, Degnim AC, et al. Expanded indications and improved outcomes for nipple-sparing mastectomy over time. Ann Surg Oncol. 2015;22(10):3317-3323. doi:10.1245/s10434-015-4737-3

31. Mobus V. Adjuvant dose-dense chemotherapy in breast cancer: standard of care in high-risk patients. Breast Care (Basel). 2016;11 (1):8-12. doi:10.1159/000444004

32. Zhou F, Feng B, Yu H, et al. Cisplatin prodrug-conjugated gold nanocluster for fluorescence imaging and targeted therapy of the breast cancer. Theranostics. 2016;6(5):679-687. doi:10.7150/thno.14556

33. Cabourne E, Clarke JC, Schlottmann PG, Evans JR. Mitomycin $\mathrm{C}$ versus 5-Fluorouracil for wound healing in glaucoma surgery. Cochrane Database Syst Rev. 2015;11:Cd006259.

34. Benada J, Macurek L. Targeting the checkpoint to kill cancer cells. Biomolecules. 2015;5(3):1912-1937. doi:10.3390/biom5031912

35. Oh E, Chim H, Soltanian HT. The effects of neoadjuvant and adjuvant chemotherapy on the surgical outcomes of breast reconstruction. J Plast Reconstr Aesthet Surg. 2012;65(10):e267-e280. doi:10.1016/ j.bjps.2012.04.053

36. Althumairi AA, Canner JK, Gearhart SL, Safar B, Sacks J, Efron JE. Predictors of perineal wound complications and prolonged time to perineal wound healing after abdominoperineal resection. World J Surg. 2016;40(7):1755-1762. doi:10.1007/s00268-016-3450-0

37. Dolen UC, Schmidt AC, Um GT, et al. Impact of neoadjuvant and adjuvant chemotherapy on immediate tissue expander breast reconstruction. Ann Surg Oncol. 2016;23(7):2357-2366. doi:10.1245/ s10434-016-5162-y

38. Choron RL, Chang S, Khan S, et al. Paclitaxel impairs adipose stem cell proliferation and differentiation. J Surg Res. 2015;196 (2):404-415. doi:10.1016/j.jss.2015.03.026

\section{Publish your work in this journal}

Cancer Management and Research is an international, peer-reviewed open access journal focusing on cancer research and the optimal use of preventative and integrated treatment interventions to achieve improved outcomes, enhanced survival and quality of life for the cancer patient.
The manuscript management system is completely online and includes a very quick and fair peer-review system, which is all easy to use. Visit http://www.dovepress.com/testimonials.php to read real quotes from published authors. 